\title{
Dense plasma heating by crossing relativistic electron beams
}

\author{
N. Ratan, ${ }^{1}$ N.J. Sircombe, ${ }^{2,1,3}$ L. Ceurvorst,${ }^{1}$ J. Sadler, ${ }^{1}$ M.F. Kasim, ${ }^{4}$ \\ J. Holloway ${ }^{4}$ M.C. Levy, ${ }^{1}$ R. Trines,${ }^{5}$ R. Bingham,${ }^{5,6}$ and P.A. Norreys ${ }^{1,5}$ \\ ${ }^{1}$ Department of Physics, University of Oxford, Parks Road, Oxford, OX1 3PU, UK \\ ${ }^{2} A W E$ plc. Aldermaston, Reading, Berkshire, RG7 $4 P R, U K$ \\ ${ }^{3}$ Department of Physics, University of Warwick, Coventry, CV4 7AL, UK \\ ${ }^{4}$ John Adams Insititute, Denys Wilkinson Building, Keble Road, Oxford OX1 3RH, UK \\ ${ }^{5}$ STFC Rutherford Appleton Laboratory, Chilton, Didcot, OX11 0QX, UK \\ ${ }^{6}$ Department of Physics, University of Strathclyde, Glasgow, G4 ONG, UK
}

(Dated: June 2, 2016)

\begin{abstract}
Here we investigate, using relativistic fluid theory and Vlasov-Maxwell simulations, the local heating of a dense plasma by two crossing electron beams. Heating occurs as an instability of the electron beams drives Langmuir waves which couple nonlinearly into damped ion-acoustic waves. Simulations show a factor 2.8 increase in electron kinetic energy with a coupling efficiency of $18 \%$. Our results support applications to the production of warm dense matter and as a driver for inertial fusion plasmas.
\end{abstract}

\section{INTRODUCTION}

Plasma heating by electron beams is the subject of intense study due to its varied applications, such as warm dense matter production [1], laboratory astrophysics [2], and magnetic and inertial confinement fusion [3, 4], and the insights into fundamental plasma theory it provides. These electron beams can be produced by the absorption of a high power laser pulse by an overdense material [5]. While beam electrons may give up their energy simply by colliding with particles in the plasma [6-9], the collective process of beam-plasma instability, in which the beam electrons act together to drive plasma waves, can also efficiently extract energy from the beam [10]. Further, this collisionless stopping process may be effective even for relativistic electron beams whose collisional stopping length is much longer than the target. On a coarse level, we might expect collisionless stopping (a collective effect) to be relevant for plasmas with a large plasma parameter [11]. Even for densities well above solid density the plasma parameter may be large at sufficiently high temperatures. In particular, the plasma parameter is large for the conditions in the hot spot of an inertial confinement fusion isobaric compression, but not in the dense fuel layer surrounding the hot spot (due to the relatively low temperature of the dense fuel). Representative inertial confinement hot spot and fuel parameters may be found in [12-14].

An analytic treatment of the collective stopping of a single relativistic electron beam was given in [15], calculating the parameters which favor collective stopping and examining strategies to localize the energy deposition. Other studies include theoretical treatments of beamplasma instabilities $[16,17]$, collective stopping of electron beams [18-20], Vlasov-Poisson simulations of collective heating [21] and ion-acoustic wave decay [22], as well as experiments on nonlinear coupling between plasma waves [23] and on plasma heating by counter-propagating laser-generated electron beams [24, 25]. Recent reviews of laser-plasma interaction [26] and fast electron transport [9] contain much detailed information together with further references.

In this paper we use relativistic multi-fluid theory (section II) and Vlasov-Maxwell simulations (section III) to consider a new mechanism by which two obliquely crossing electron beams can efficiently transfer their energy to a dense plasma. Specifically, we show an instability in which the crossing beams drive Langmuir waves in the plasma, and these Langmuir waves nonlinearly couple into large-amplitude ion-acoustic waves. These ionacoustic waves decay leading to ion-acoustic turbulence. We demonstrate that the background electrons show a factor 2.8 increase in kinetic energy with a coupling efficiency of $18 \%$.

\section{LINEAR THEORY}

To investigate the first step of the energy cascade process, we derive the linear growth rates for the instability of two obliquely crossing relativistic electron beams in a background plasma. Here the two beams and the background plasma are each described by a separate relativistic fluid, giving three fluids in total, and the ions provide a stationary neutralizing background of density $n_{0}$ (note that because the ions here are fixed, all modes in Fig. 1 are electron modes, as opposed to the simulations below where ions are free to move). The equations governing the system are Maxwell's equations and continuity and momentum equations for each electron fluid, together with equations defining the charge and current densities $\rho$ and $\boldsymbol{J}$ in terms of the fluid densities and ve- 
locities $n_{\alpha}$ and $\boldsymbol{v}_{\alpha}(\alpha=1,2,3)$,

$$
\begin{aligned}
& \vec{\nabla} \cdot \vec{E}=\frac{1}{\epsilon_{0}} \rho \\
& \vec{\nabla} \times \vec{B}-\frac{1}{c^{2}} \partial_{t} \vec{E}=\mu_{0} \vec{J} \\
& \vec{\nabla} \times \vec{E}+\partial_{t} \vec{B}=0 \\
& \vec{\nabla} \cdot \vec{B}=0 \\
& \partial_{t} n_{\alpha}+\vec{\nabla} \cdot\left(n_{\alpha} \overrightarrow{v_{\alpha}}\right)=0 \\
& \left(\partial_{t}+\overrightarrow{v_{\alpha}} \cdot \vec{\nabla}\right) \gamma_{\alpha} \vec{v}_{\alpha}=\frac{q}{m}\left(\vec{E}+\overrightarrow{v_{\alpha}} \times \vec{B}\right) \\
& \vec{J}=q n_{1} \overrightarrow{v_{1}}+q n_{2} \overrightarrow{v_{2}}+q n_{3} \overrightarrow{v_{3}} \\
& \rho=q n_{1}+q n_{2}+q n_{3}-q n_{0}
\end{aligned}
$$

where $n_{1}, n_{2}, \overrightarrow{v_{1}}$ and $\overrightarrow{v_{2}}$ are the beams' number densities and velocities, $n_{3}$ and $\overrightarrow{v_{3}}$ are the background plasma number density and velocity, $\gamma_{j}=\left(1-v_{j}^{2} / c^{2}\right)^{-\frac{1}{2}}, q$ is the electron charge, $m$ is the electron mass, and $n_{0}$ is the background ion density (here we assume singly charged ions). The instability growth rates may be calculated using standard linearization methods [11] in which the field equations are reduced to a linear equation

$$
M(\boldsymbol{k}, \omega) \boldsymbol{u}=0
$$

where if $N$ is the number of fields then $M$ is an $N \times$ $N$ matrix depending on wavevector $\boldsymbol{k}$ and frequency $\omega$ and $\boldsymbol{u}$ is the $N$ element vector of field mode amplitudes. The explicit form of this dispersion matrix $M$ is given in the appendix. The frequencies of modes with a given wavevector $\boldsymbol{k}$ are then determined from the dispersion relation

$$
\operatorname{det}(M(\boldsymbol{k}, \omega))=0 .
$$

Fig. 1 shows the theoretical growth rate maps of the instability for $1 \mathrm{MeV}$ electron beams with a density, $n_{b}$, of 0.01 times the background density, $n_{0}$, on the top row $(\mathrm{a}, \mathrm{b})$, and for $0.1 \mathrm{MeV}$ electron beams with $n_{b} / n_{0}=0.1$ on the bottom row $(\mathrm{c}, \mathrm{d})$. The left column $(\mathrm{a}, \mathrm{c})$ corresponds to beams crossing at $90^{\circ}$, travelling in the positive $x$ and $y$ directions. The right column $(\mathrm{b}, \mathrm{d})$ corresponds to beams crossing at $180^{\circ}$ travelling along the $x$ axis. The system of two beams crossing at $90^{\circ}$ has a symmetry about the line $y=x$ which induces the symmetry of the growth rate maps in (a) and (c) under reflection in the line $k_{y}=k_{x}$. With the beams crossing at $180^{\circ}$ the system has a symmetry under reflection in the $x$ and $y$ axes which is again inherited by the growth rate map. Note that the effectiveness of the second stage of the energy cascade, that is, the nonlinear coupling of the Langmuir waves into ion-acoustic waves, will depend on the wavevector spectrum of the Langmuir waves, since the nonlinear coupling strength depends on the relative orientations of the wavevectors involved [18]. The cases (a)
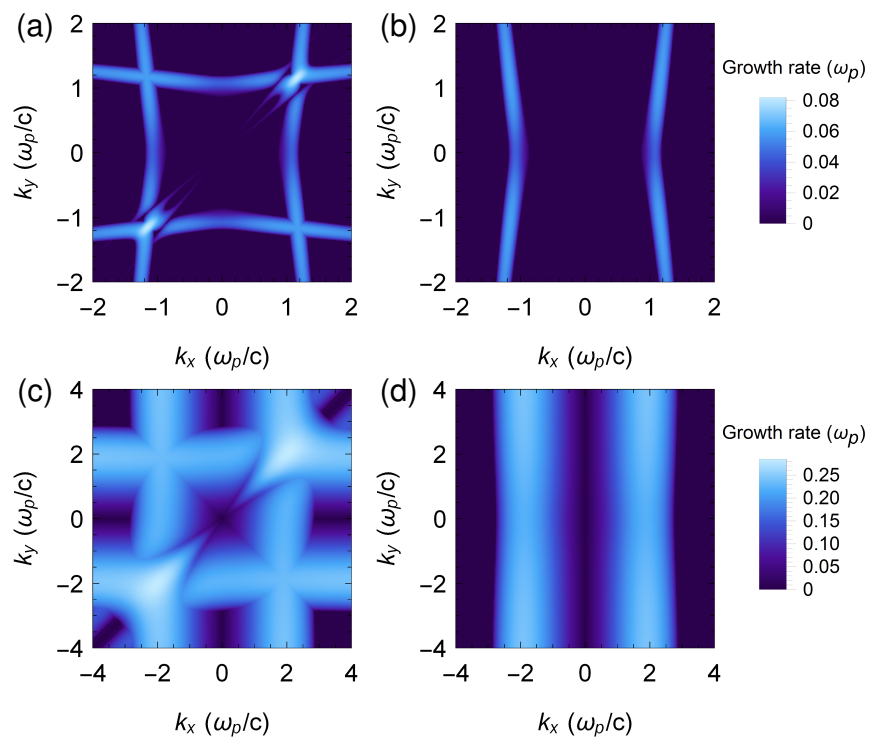

FIG. 1. Theoretical growth rate maps for the first stage of the energy cascade process: the instability arising due to two beams crossing in a dense plasma. The growth rates are shown for beams crossing at an angle of $90^{\circ}$ in (a) and (c) and $180^{\circ}$ in (b) and (d). The beam energy $E$ and beam density fraction $n_{b} / n_{0}$ are $E=1 \mathrm{MeV}, n_{b} / n_{0}=0.01$ in (a) and (b), $E=0.1 \mathrm{MeV}, n_{b} / n_{0}=0.1$ in (c) and (d). Note the different scales.

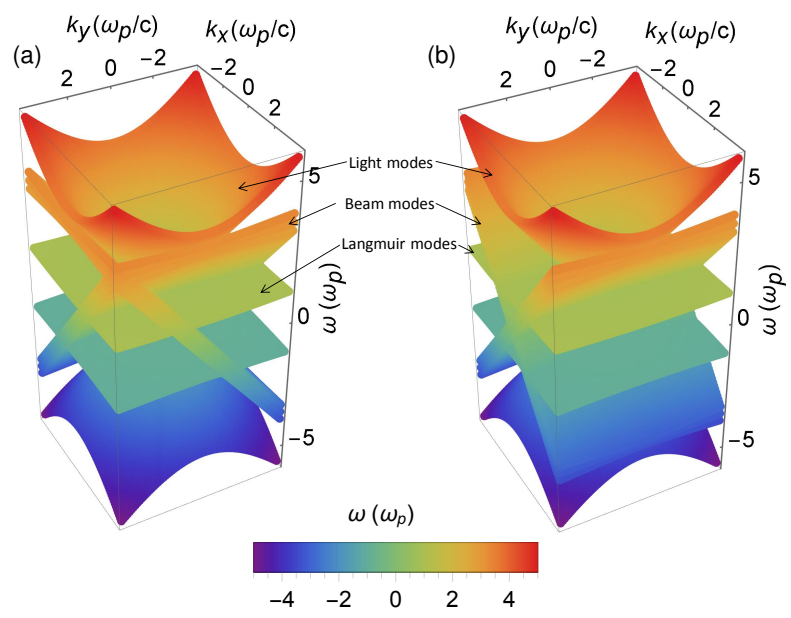

FIG. 2. The real part of the mode frequencies for a system of two relativistic electron beams crossing in a background plasma at angles of (a) $90^{\circ}$ and (b) $180^{\circ}$. The different sheets of the dispersion surface correspond to different modes of the system.

and (c) in which the beams cross at $90^{\circ}$ give a higher maximum growth rate, corresponding to a mode with $k_{x}=k_{y}$ travelling diagonally between the two beams, as well as a spectrum of unstable modes which covers a wider range of angles. Since in the linear phase of the instability each mode grows independently, these growth 

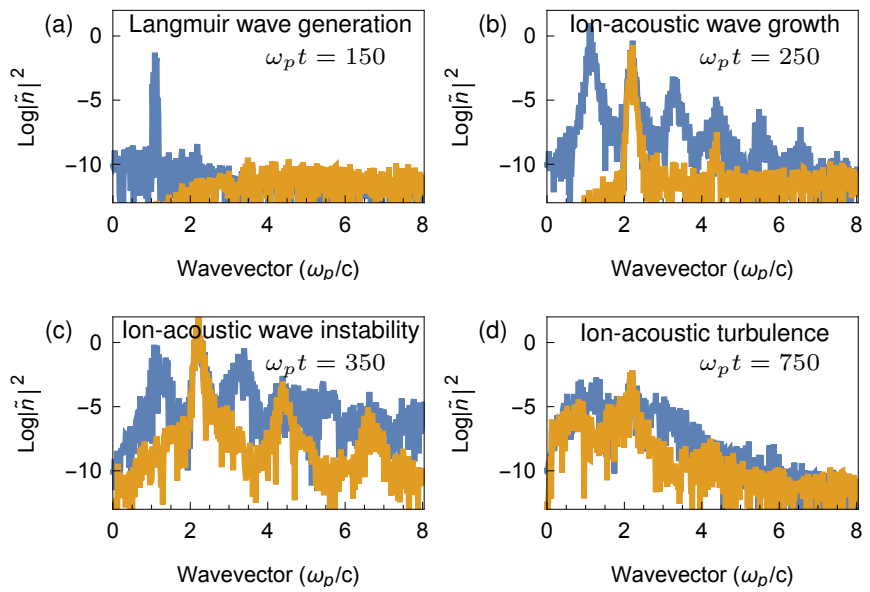

Electrons Ions

FIG. 3. Time history of the power spectra of the electron and ion densities from a 1D Vlasov simulation of two electron beams crossing at $180^{\circ}$, showing the waves present during each stage of the heating process. Each timestep shows a different stage of the energy cascade: (a) the generation of Langmuir waves by the instability of the crossing beams, (b) the nonlinear coupling of the Langmuir waves into ion waves, (c) the instability of the ion-acoustic waves and (d) ion-acoustic turbulence.

rate maps show that beams crossing at $90^{\circ}$ will initially couple their energy into Langmuir waves more rapidly.

Fig. 2 shows the real part of the frequencies which solve the $2 \mathrm{D}$ dispersion relation as a function of $2 \mathrm{D}$ wavevector $\vec{k}$. The different sheets of the dispersion surface correspond to different modes of the system. In particular the diagonal quasi-planar sheets correspond to beam modes propagating at the beam velocities. The curved sheets at the top and bottom are light modes. Only a small number of the modes shown here are unstable, that is, have a positive imaginary component of frequency.

\section{VLASOV-MAXWELL SIMULATIONS}

To follow the nonlinear evolution of the crossing beams and the coupling of the beam-driven Langmuir waves into ion-acoustic waves, we have performed simulations with the Vlasov-Maxwell code VALIS [27]. This code solves the Vlasov-Maxwell system in up to two space and two momentum dimensions on an Eulerian phase space grid. VALIS advances the distribution functions from one time step to the next using an operator-split conservative scheme with separate $1 \mathrm{D}$ advections in each position and momentum coordinate [28], the advections being performed using the Piecewise Parabolic Method [29]. A Vlasov code is well suited to the present study due to its low noise and ability to resolve the distribution function $f$ even in regions of phase space where $f$ is very small, both very useful traits for studying insta- bilities. We consider electron beams crossing at $180^{\circ}$ in a background plasma. The background electron and ion temperatures are $4 \mathrm{keV}$, corresponding to the range of temperatures of hot spots in inertial confinement fusion targets. The background plasma density in the simulation is a free parameter setting the scale of the units, though we have in mind an electron number density of $n=10^{26} \mathrm{~cm}^{-3}$. Both electron beams have a mean relativistic kinetic energy of $1 \mathrm{MeV}$ and a density of one percent of the background density, in the practically relevant regime of high beam energy and low fractional beam density. The two beam distributions have a temperature of $40 \mathrm{keV}$. The narrow energy spread of the beams used here deserves comment; while the electron energy distribution generated by a typical overdense laser-plasma interaction is often characterized by one or two components with exponential decay in energy, it is conceivable that during the transport of the beam through dense matter the lower energy electrons will be significantly stopped, leaving the remaining distribution with a narrower peak in energy [30]. No collision operator is used since for a plasma of number density $n=10^{26} \mathrm{~cm}^{-3}$ and electron and ion temperatures $T_{\mathrm{e}}=T_{\mathrm{i}}=4 \mathrm{keV}$, the electron plasma frequency is $\omega_{p e}=5.64 \times 10^{17} \mathrm{~s}^{-1}$, over 200 times the electron collision frequency, while the simulations here ran for a time $2000 / \omega_{p e}$ corresponding to 320 plasma periods, with much of the interesting dynamics occurring early in the simulation.

The initial electron distribution function is a sum of three drifting Maxwell-Jüttner distributions. The distributions are initially homogeneous and periodic boundary conditions are employed. Due to the low noise level in the code an initial low amplitude white noise perturbation is included in the electrons' spatial distribution to seed the initial beam-plasma instability. We simulate the system in one space and one momentum dimension, and hence waves which would propagate obliquely to the beams cannot be captured. The ion mass in the simulations is $m_{i}=1000 m_{e}$. While this ion mass is only 0.54 times the mass of a hydrogen ion (or 0.27 times the mass of a deuterium ion), we do not expect this to change the results significantly since with $m_{i}=1000 m_{e}$ the time scale of the natural oscillations of the ions is an order of magnitude greater than the electrons and so the ion population will evolve on a different timescale to the electrons. The spatial grid covers $400 c / \omega_{p 0}$ and the time $2000 / \omega_{p 0}$. The relativistic momentum space for the electrons spans $\pm 20 m_{e} c$, for the ions $\pm 0.25 m_{i} c$. The dimension of the $(x, p)$ phase space grid was $4196 \times 2048$ for both electrons and ions.

Fig. 3 shows a time history of the power spectra of the electron and ion densities (the modulus squared of their spatial Fourier transforms). Each frame shows a different stage of the energy cascade. In (a) at $\omega_{p} t=150$ there is a single peak in the electron density spectrum representing a Langmuir wave driven by the two beams crossing in the plasma. It is at a wavevector $k_{0}=1.1 \omega_{p} / c$, in agreement with the most unstable mode predicted by the multiple 

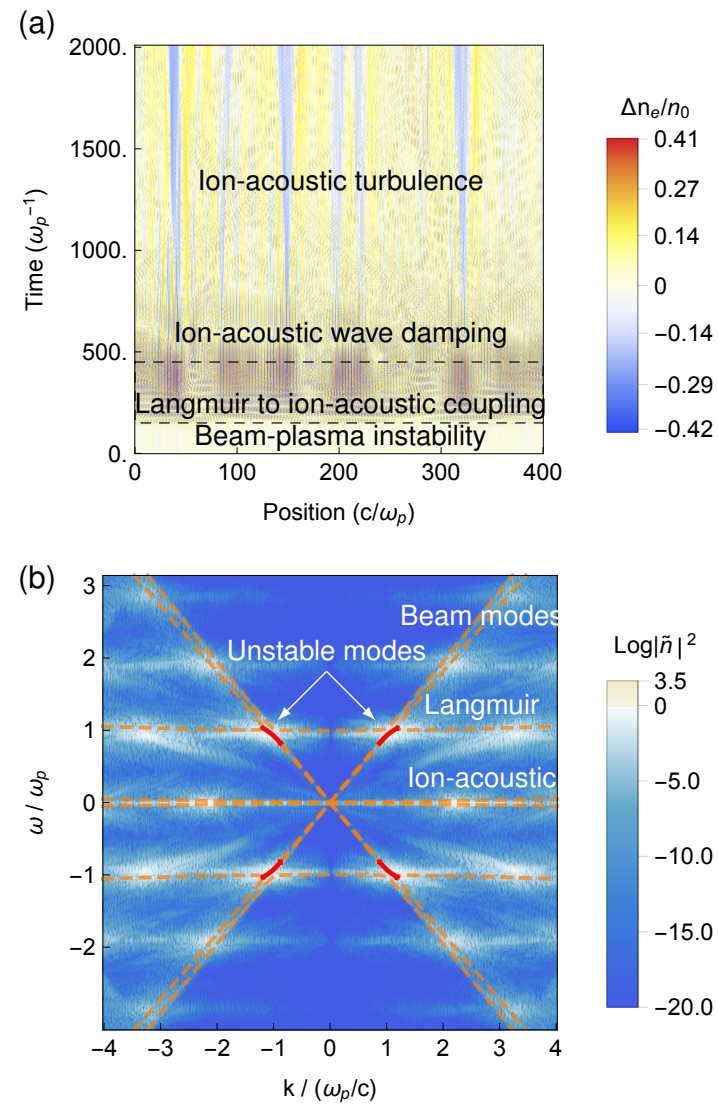

FIG. 4. (a) Space-time plot of the electron density showing each stage of the energy cascade. (b) The numerically calculated frequency and wavevector, $\omega$ and $k$, of the waves produced by instability or nonlinear coupling in the dashed boxed portion of Fig. 4 (a). Overlaid are the theoretical dispersion curves. The red sections of the dispersion curves indicate frequencies with a positive imaginary part, showing instability.

relativistic fluid theory. In (b) at $\omega_{p} t=250$ we see a peak at $2 k_{0}$ in both the electron and ion spectra representing an ion-acoustic wave driven nonlinearly by the beam driven Langmuir wave [18]. The higher order peaks in the electron spectrum are Langmuir waves resulting from the nonlinear coupling. In (c) at $\omega_{p} t=350$ the ion-acoustic feature in the electron and ion spectra from (b) has generated higher order peaks as the ion-acoustic wave becomes unstable. In (d) at $\omega_{p} t=750$ sharp peaks are no longer visible in the electron and ion spectra, the continuous fall off with wavevector indicating a turbulent state.

Fig. 4 (a) shows a space-time plot of the electron density from the simulation, giving a global picture of the interaction. Initially the beams drive Langmuir waves in the plasma via beam-plasma instability. These Langmuir waves become large and couple into ion-acoustic waves. The ion-acoustic waves are then damped, and the plasma ends in a state of ion-acoustic turbulence. The bunched structures in the dashed boxed region of Fig. 4 (a) show that localized wavepackets are formed. Fig. 4 (b) shows the Fourier transform of the portion
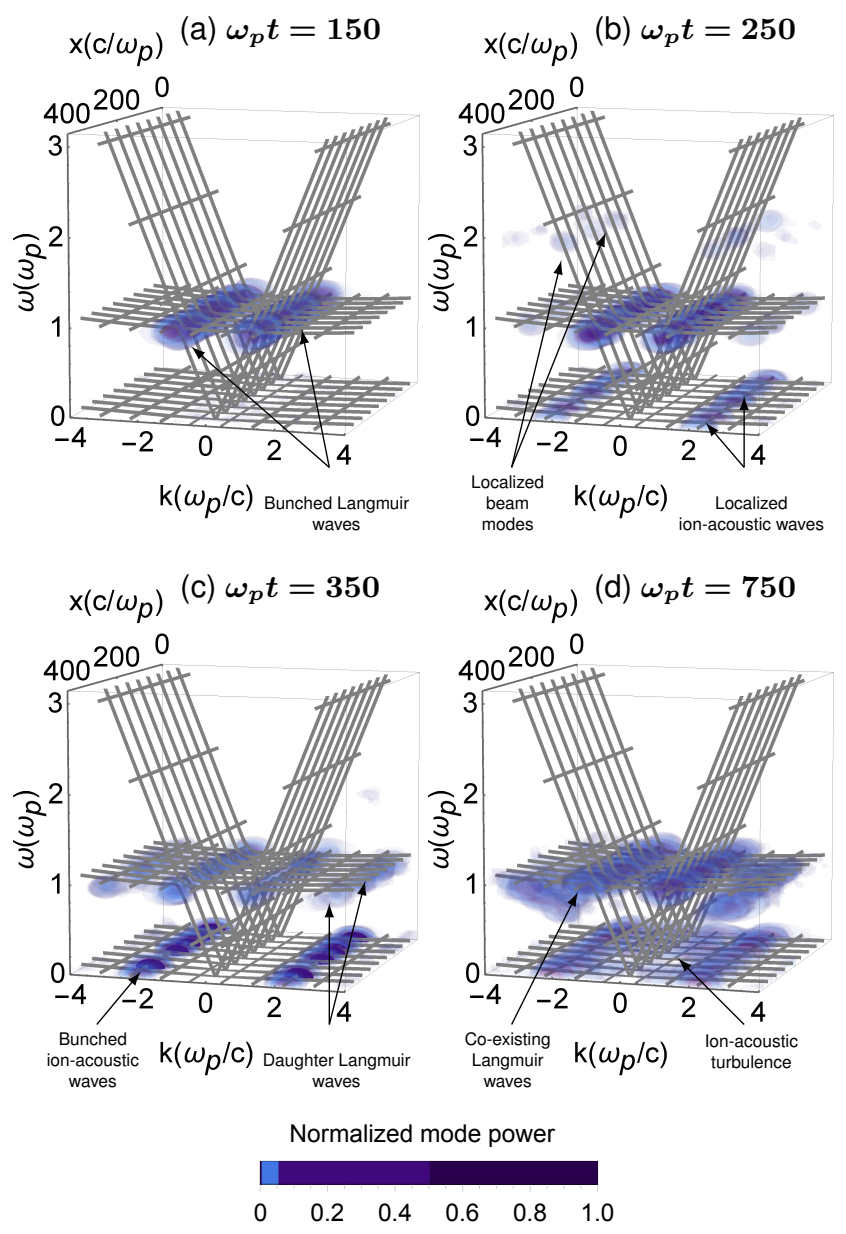

FIG. 5. Time history of the wave phase space distribution of the electron density. This shows the distribution of the plasma waves in the space of position, wavevector and frequency (see text). The gridded surfaces are the theoretical dispersion surfaces.

of Fig. 4 (a) indicated by the dashed box, highlighting the time in which the beams become unstable and ionacoustic waves begin to form. Fig. 4 (b) shows the distribution of waves in wavevector and frequency, i.e. in $(k, \omega)$ space. This provides a discrete approximation to the space-time Fourier transform $\tilde{n}(k, \omega)$ of the electron density $n(x, t), \tilde{n}(k, \omega)=\int n(x, t) \exp (-i(k x-\omega t)) \mathrm{d} x \mathrm{~d} t$. The orange curves plotted on (b) show the roots of the dispersion relation from an electrostatic relativistic multi-fluid model for the two beams, background electrons and ions, with temperature terms included. The predominance of modes along the linear dispersion curves in Fig. 4 (b) demonstrates the utility of the linear wave properties even in this nonlinear interaction. The red sections of the dispersion curves have a positive imaginary component of the frequency, leading to an exponential growth of the mode amplitude and thus instability. Fig. 4 shows the evolution of the waves responsible for the energy cascade from the electron beams to the plasma 


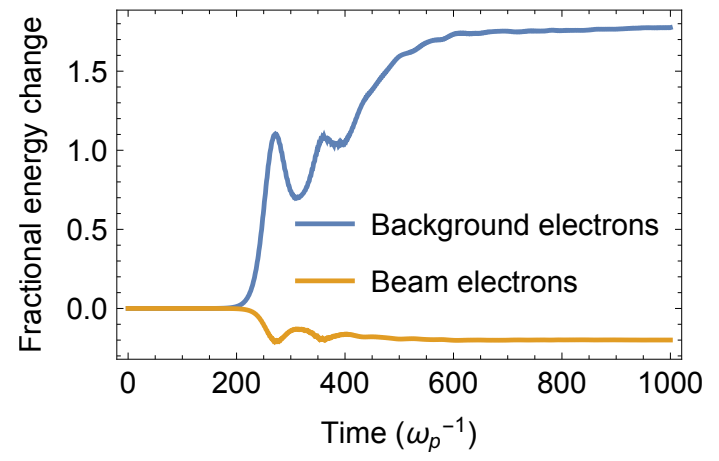

FIG. 6. Time evolution of the fractional energy change of the background electrons (blue line) and beam electrons (orange line) during the collisionless heating process.

particles.

To further study the evolution of the waves shown in Fig. 4 responsible for the energy cascade, we employ a mathematical representation which reveals detailed information on the time evolution of the wavevector, frequency and location of the waves. This is a wave phase space distribution [31, 32], here defined as a space-time windowed Fourier transform $g$ according to $g(x, k, t, \omega)=\int n(y, s) w_{x t}(y, s) \exp (-i(k y-\omega s)) \mathrm{d} y \mathrm{~d} s$, where $w_{x t}(y, s)=\exp \left(-(y-x)^{2} / \sigma_{x}^{2}-(s-t)^{2} / \sigma_{t}^{2}\right)$ is a Gaussian window function and the parameters $\sigma_{x}$ and $\sigma_{t}$ determine the window width in space and time.

Fig. 5 shows volume plots of $|g(x, k, t, \omega)|^{2}$ for a range of times $t$, which we may interpret as the distribution of waves in position, wavevector and frequency at the time $t$. The gridded surfaces show the dispersion surfaces from the relativistic multi-fluid theory used in Fig. 4. Fig. 5 (a) shows the early formation of bunched high frequency Langmuir waves, located along the Langmuir wave dispersion surface as predicted by the linear theory. (b) shows that the ion-acoustic waves generated are localized near the position of the large Langmuir wavepackets. This local information is important since the Langmuir waves are able to drive ion-acoustic waves more rapidly by bunching to increase the local Langmuir wave intensity, which in turn increases the coupling rate into ion-acoustic waves. (c) shows similarly localized daughter Langmuir waves together with bunched ion-acoustic waves, while in (d) the spread of the low frequency part of the distribution in $k$ indicates ion-acoustic turbulence. Fig. 5 unfolds the plasma dynamics into the interaction of localized wavepackets of a variety of frequencies and wavevectors, providing a detailed picture of the evolution of the waves responsible for the energy cascade process.

Fig. 6 shows the time evolution of the fractional kinetic energy change of the beam electrons and the background electrons. These energies are calculated using a simulation in which the electron beams and the background electrons are separate species, allowing a clean calculation of the energy of each component. Fig. 6 shows that $18 \%$ of the electron beam kinetic energy is coupled
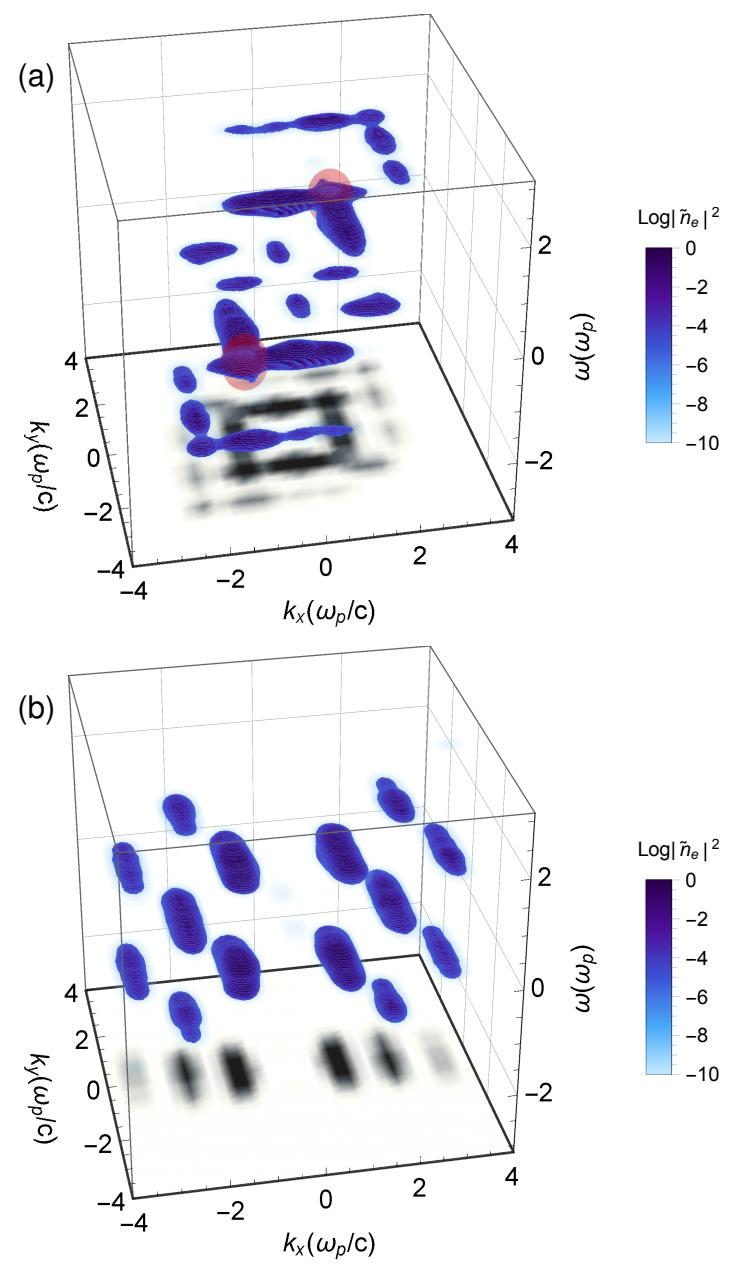

FIG. 7. Numerically calculated $\boldsymbol{\omega}-\boldsymbol{k}$ distribution of the electron density from 2D Vlasov-Maxwell simulations of $1 \mathrm{MeV}$ beams crossing at (a) $90^{\circ}$ and (b) $180^{\circ}$. The bottom faces show the distribution in wavevector $\boldsymbol{k}$ integrated over frequency $\omega$. The red spheres in (a) show the most unstable modes predicted by the relativistic multi-fluid theory of Fig. 1 (a).

into the plasma electrons, giving a factor 2.8 increase in plasma electron kinetic energy.

Fig. 7 shows the electron density distribution in $\omega$ and $\boldsymbol{k}$ from 2D Vlasov-Maxwell simulations of two $1 \mathrm{MeV}$ electron beams of density $n_{b} / n_{0}=0.01$ crossing in a plasma at (a) $90^{\circ}$ and (b) $180^{\circ}$. The largest amplitude wave in (a) travels diagonally between the beams with positive projection of its phase velocity vector along each beam velocity vector. It occurs at the wavevector and frequency predicted by the linear theory above, shown in (a) by the red spheres. Note that the modes corresponding to the two red spheres have phase velocities in the same direction although their wavevectors point in opposite directions, since their frequencies differ by a factor of -1 . Both positive and negative frequencies are shown here since including the negative frequency modes facilitates the consideration of nonlinear wave coupling. The bases 
of the $3 \mathrm{D}$ plots show the wave distribution in $(\omega, \vec{k})$ integrated over $\omega$, giving the distribution in $\vec{k}$ space which is here two-dimensional.

\section{CONCLUSIONS}

In conclusion, we have provided a theoretical and computational study of dense plasma heating using crossing relativistic electron beams. The heating is due to the collective processes of beam-plasma instabilities and nonlinear wave coupling. Using a relativistic multi-fluid model we have calculated linear growth rates for the instability of two electron beams obliquely crossing in a plasma. Vlasov-Maxwell simulations exhibit features predicted by the theory for the beam-plasma instability and the subsequent nonlinear coupling of Langmuir and ion-acoustic waves. Using a wave phase space analysis we have studied the evolution of the location, wavenumber and frequency of all waves involved in the heating process in clear relief. The simulations show a kinetic to thermal coupling efficiency of $18 \%$ into the plasma electrons giving a factor of 2.8 increase in plasma electron kinetic energy. Further, the effect of collisions on a longer timescale will act to thermalize the background electron distribution and equalize the electron and ion temperatures on a timescale of $\sim 5$ picoseconds. This means that within the confinement time of an inertial-fusion target some of the energy collectively coupled into the background electrons may be transferred to the plasma ions and so contribute to increasing the neutron yield. These results are very encouraging for the auxiliary heating concept [33], where the heating of the central hot spot by shock wave convergence $[12,34]$ is supplemented by this process.

\section{ACKNOWLEDGMENTS}

The authors thank all of the staff of the Central Laser Facility and the Scientific Computing Department at STFC Rutherford Appleton Laboratory for their help and support. This work has been carried out within the framework of the EUROfusion Consortium and has received funding from the Euratom research and training programme 2014-2018 under grant agreement No 633053. Computing resources provided by STFC Scientific Computing Department's SCARF cluster. The authors would like to acknowledge the use of the University of Oxford Advanced Research Computing (ARC) facility in carrying out this work [35]. MCL thanks the Royal Society Newton International Fellowship for funding. This work was also supported by OxCHEDS, STFC and EPSRC. PAN gratefully acknowledges the William Penney Fellowship at AWE plc.

\section{APPENDIX}

In this appendix we show the explicit form of the dispersion matrix $M(\boldsymbol{k}, \omega)$ used in the linear theory section to derive the frequencies and growth rates from our relativistic multi-fluid model. Since $M$ is an $18 \times 18$ matrix we have displayed it as four blocks $A, B, C$, and $D$ in terms of which $M=(A, B, C, D)$. Here the quantity $\beta_{\alpha j}=v_{\alpha j} / c$ is the $j^{\text {th }}$ component of the velocity of species $\alpha$ normalized to the speed of light and $n_{\alpha}$ is the zeroth order density of species $\alpha$ normalized to the background ion density.

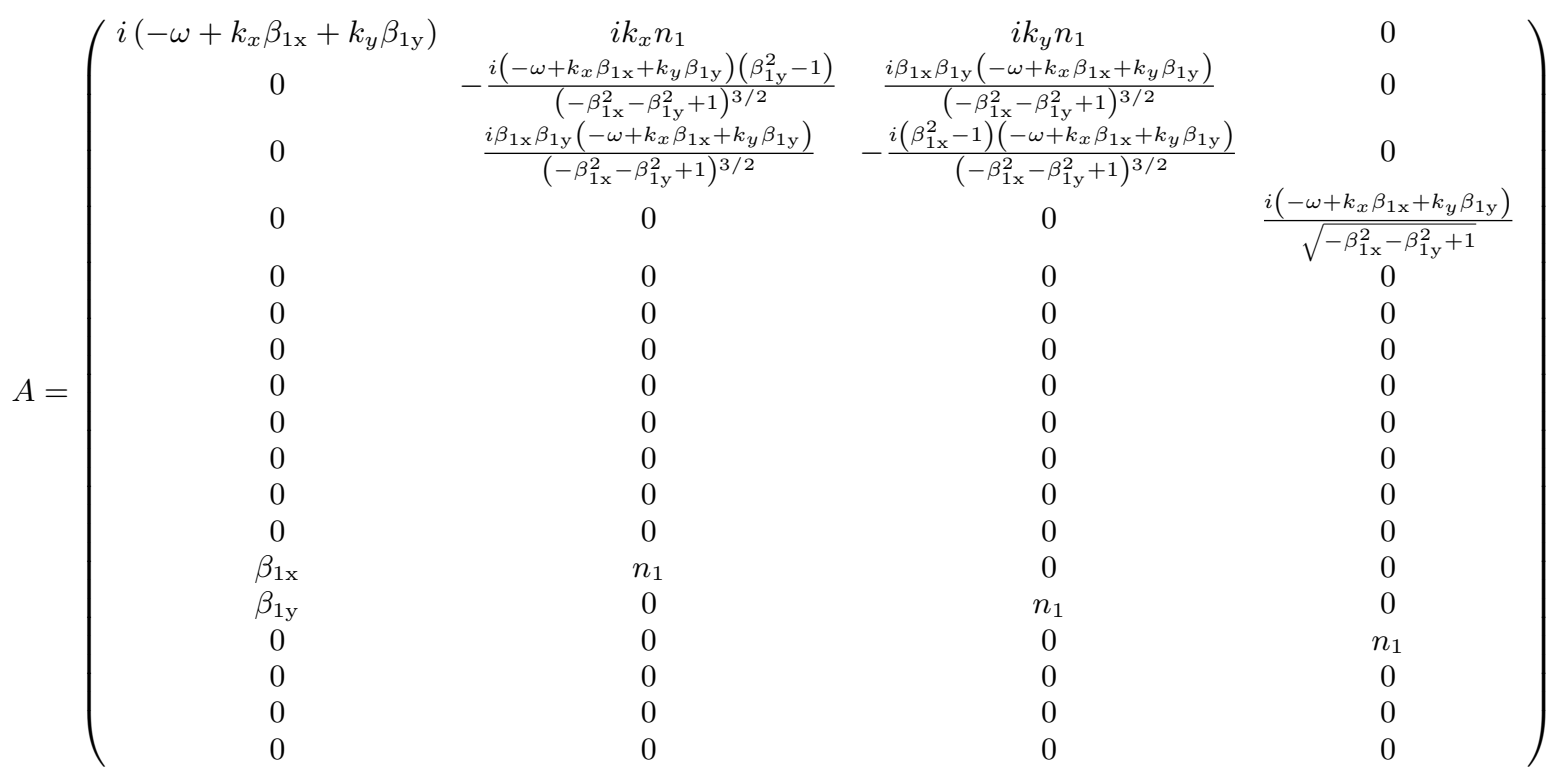




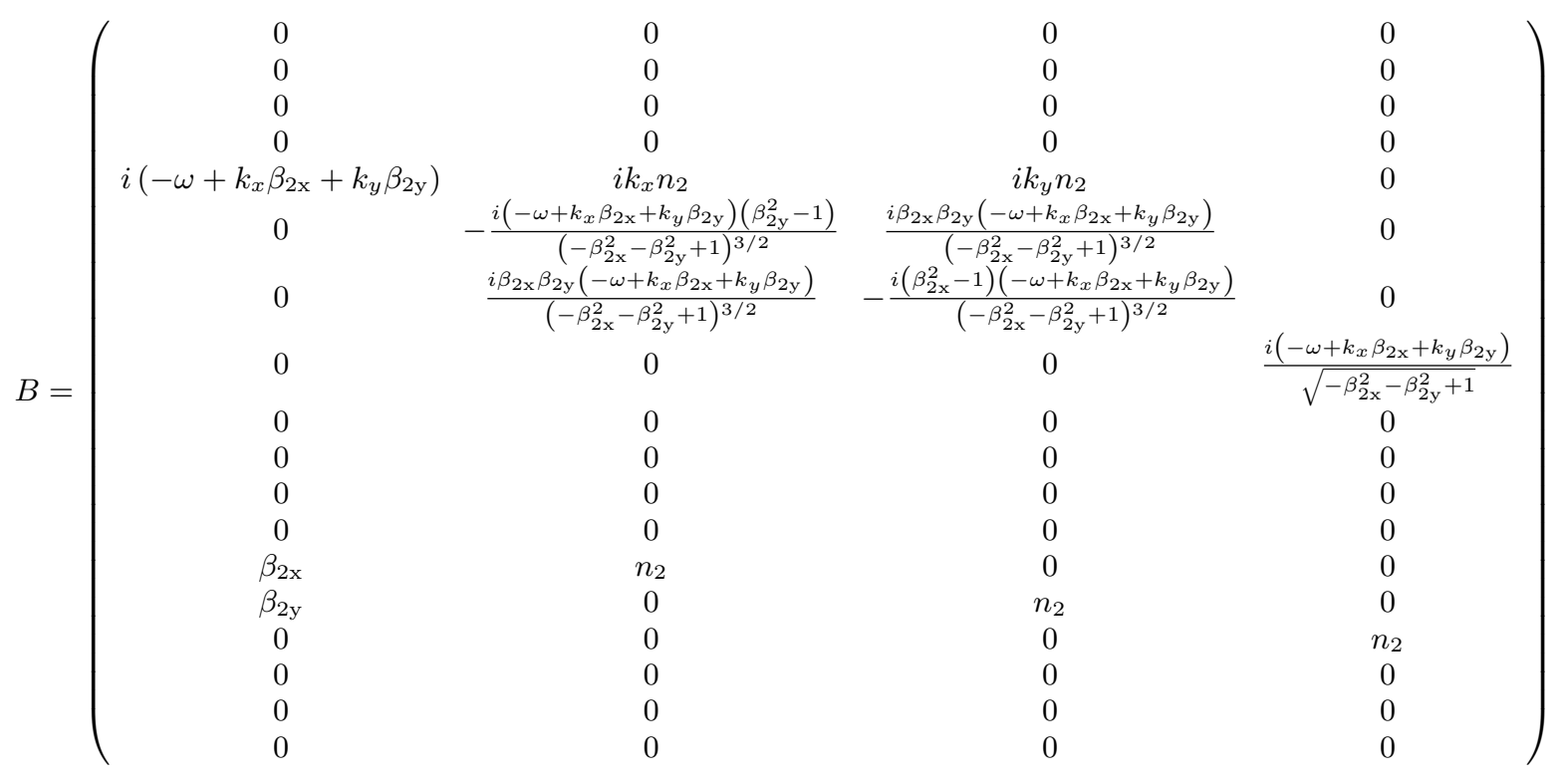

(12)

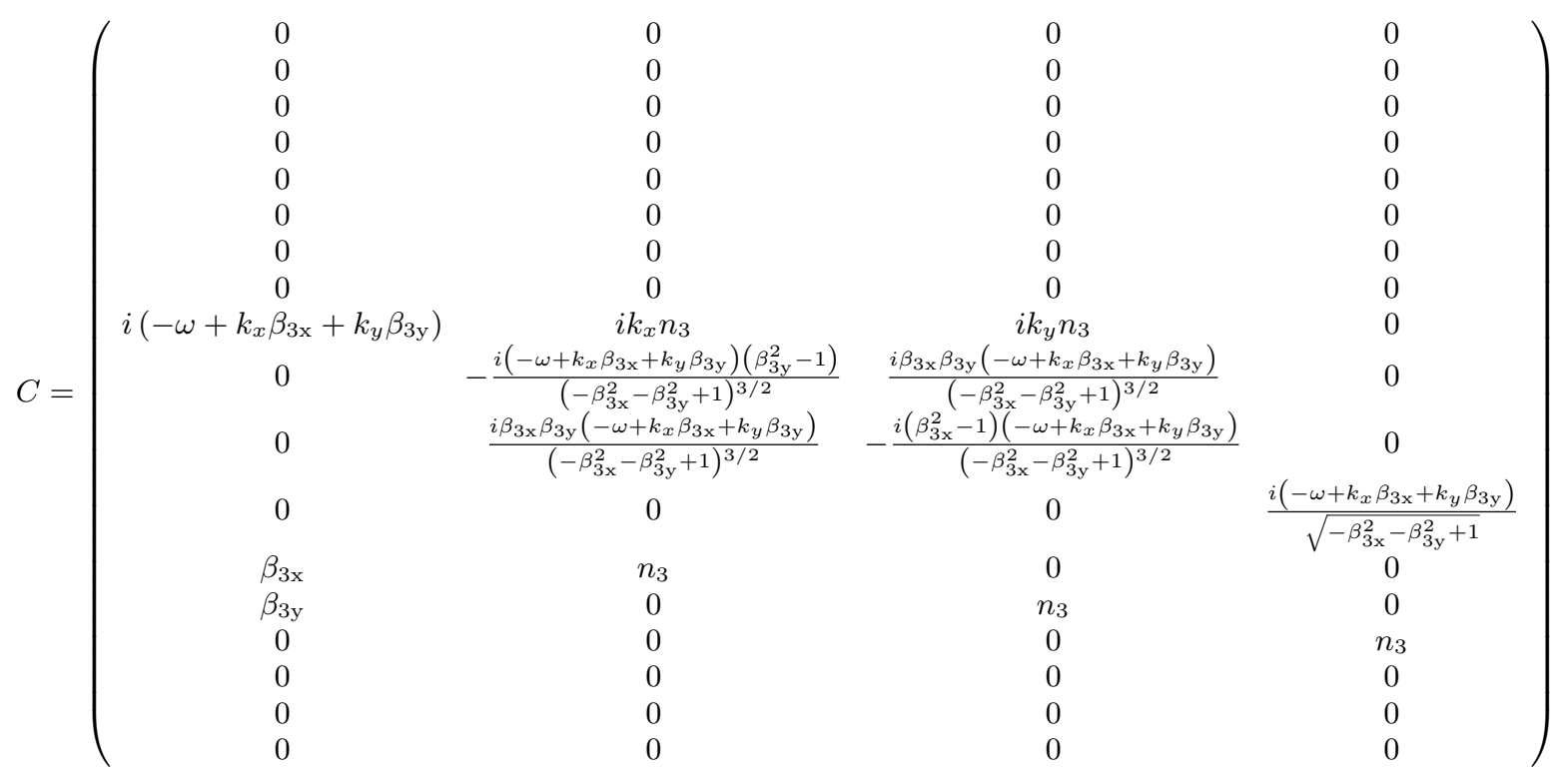

$$
D=\left(\begin{array}{cccccc}
0 & 0 & 0 & 0 & 0 & 0 \\
1 & 0 & 0 & 0 & 0 & 0 \\
0 & 1 & 0 & 0 & 0 & 0 \\
0 & 0 & 1 & 0 & 0 & 0 \\
0 & 0 & 0 & 0 & 0 & 0 \\
1 & 0 & 0 & 0 & 0 & 0 \\
0 & 1 & 0 & 0 & 0 & 0 \\
0 & 0 & 1 & 0 & 0 & 0 \\
0 & 0 & 0 & 0 & 0 & 0 \\
1 & 0 & 0 & 0 & 0 & 0 \\
0 & 1 & 0 & 0 & 0 & 0 \\
0 & 0 & 1 & 0 & 0 & 0 \\
i \omega & 0 & 0 & 0 & 0 & i k_{y} \\
0 & i \omega & 0 & 0 & 0 & -i k_{x} \\
0 & 0 & i \omega & -i k_{y} & i k_{x} & 0 \\
0 & 0 & i k_{y} & -i \omega & 0 & 0 \\
0 & 0 & -i k_{x} & 0 & -i \omega & 0 \\
-i k_{y} & i k_{x} & 0 & 0 & 0 & -i \omega
\end{array}\right)
$$


[1] D. J. Hoarty, S. F. James, H. Davies, C. R. D. Brown, J. W. O. Harris, C. C. Smith, S. J. Davidson, E. Kerswill, B. J. B. Crowley, and S. J. Rose, High Energy Density Physics 3, 115 (2007).

[2] G. Sarri, K. Poder, J. M. Cole, W. Schumaker, A. Di Piazza, B. Reville, T. Dzelzainis, D. Doria, L. A. Gizzi, G. Grittani, et al., Nature communications 6 (2015).

[3] M. Tabak, J. Hammer, M. E. Glinsky, W. L. Kruer, S. C. Wilks, J. Woodworth, E. M. Campbell, M. D. Perry, and R. J. Mason, Physics of Plasmas (1994-present) 1, 1626 (1994).

[4] H. Shiraga, H. Nagatomo, W. Theobald, A. A. Solodov, and M. Tabak, Nuclear Fusion 54, 054005 (2014).

[5] M. C. Levy, S. C. Wilks, M. Tabak, S. B. Libby, and M. G. Baring, Nature communications 5 (2014).

[6] International Commission on Radiation Units and Measurements, ICRU Report 37, Stopping Powers for Electrons and Positrons (Bethesda, MD, 1984).

[7] S. Atzeni, A. Schiavi, and J. Davies, Plasma Physics and Controlled Fusion 51, 015016 (2008).

[8] A. Solodov and R. Betti, Physics of Plasmas (1994present) 15, 042707 (2008).

[9] A. P. L. Robinson, D. J. Strozzi, J. R. Davies, L. Gremillet, J. J. Honrubia, T. Johzaki, R. J. Kingham, M. Sherlock, and A. A. Solodov, Nuclear Fusion 54, 054003 (2014).

[10] O. Buneman, Physical Review 115, 503 (1959).

[11] D. R. Nicholson, Introduction to Plasma Theory (Wiley, 1983).

[12] O. A. Hurricane, D. A. Callahan, D. T. Casey, P. M. Celliers, C. Cerjan, E. L. Dewald, T. R. Dittrich, T. Döppner, D. E. Hinkel, L. F. B. Hopkins, et al., Nature (2014).

[13] J. Milovich, H. Robey, D. Clark, K. Baker, D. Casey, C. Cerjan, J. Field, A. MacPhee, A. Pak, P. Patel, et al., Physics of Plasmas (1994-present) 22, 122702 (2015).

[14] O. Hurricane, D. Callahan, D. Casey, E. Dewald, T. Dittrich, T. Döppner, S. Haan, D. Hinkel, L. B. Hopkins, O. Jones, et al., Nature Physics (2016).

[15] V. M. Malkin and N. J. Fisch, Physical Review Letters 89, 125004 (2002).

[16] S. A. Bludman, K. M. Watson, and M. N. Rosenbluth, Physics of Fluids (1958-1988) 3, 747 (1960).

[17] A. Bret, L. Gremillet, and M. E. Dieckmann, Physics of Plasmas (1994-present) 17, 120501 (2010).
[18] V. N. Tsytovich, Nonlinear Effects in Plasma (Plenum, 1970).

[19] T. Tajima, Journal of Plasma Physics 19, 63 (1978).

[20] J. T. Mendonça, P. Norreys, R. Bingham, and J. R. Davies, Physical Review Letters 94, 245002 (2005).

[21] N. J. Sircombe, R. Bingham, M. Sherlock, J. T. Mendonca, and P. Norreys, Plasma Physics and Controlled Fusion 50, 065005 (2008).

[22] T. Chapman, S. Brunner, J. W. Banks, R. L. Berger, B. I. Cohen, and E. A. Williams, Physics of Plasmas (1994-present) 21, 042107 (2014).

[23] M. J. Everett, A. Lal, C. E. Clayton, W. B. Mori, T. W. Johnston, and C. Joshi, Physical Review Letters 74, 2236 (1995).

[24] Y. Kitagawa, Y. Mori, O. Komeda, K. Ishii, R. Hanayama, K. Fujita, S. Okihara, T. Sekine, N. Satoh, T. Kurita, et al., Physical Review Letters 108, 155001 (2012).

[25] P. Koester, N. Booth, C. A. Cecchetti, H. Chen, R. G. Evans, G. Gregori, L. Labate, T. Levato, B. Li, M. Makita, et al., Physics of Plasmas (1994-present) 22, 020701 (2015).

[26] A. J. Kemp, F. Fiuza, A. Debayle, T. Johzaki, W. B. Mori, P. K. Patel, Y. Sentoku, and L. O. Silva, Nuclear Fusion 54, 054002 (2014).

[27] N. J. Sircombe and T. D. Arber, Journal of Computational Physics 228, 4773 (2009).

[28] C.-Z. Cheng and G. Knorr, Journal of Computational Physics 22, 330 (1976).

[29] P. Colella and P. R. Woodward, Journal of Computational Physics 54, 174 (1984).

[30] J. M. Hill, M. H. Key, S. P. Hatchett, and R. R. Freeman, Physics of Plasmas (1994-present) 12, 082304 (2005).

[31] S. W. McDonald, Physics Reports 158, 337 (1988).

[32] K. Rönnmark and J. Larsson, Journal of Geophysical Research: Space Physics (1978-2012) 93, 1809 (1988).

[33] P. Norreys, in APS Meeting Abstracts, Vol. 1 (2014) p. 6011.

[34] H.-S. Park, O. A. Hurricane, D. A. Callahan, D. T. Casey, E. L. Dewald, T. R. Dittrich, T. Döppner, D. E. Hinkel, L. F. B. Hopkins, S. Le Pape, et al., Physical Review Letters 112, 055001 (2014).

[35] A. Richards, University of Oxford Advanced Research Computing (2015) zenodo.10.5281/zenodo.22558. 